\title{
Análise biométrica de frutos e sementes de Passiflora cristalina Vanderplank \& Zappi
}

\author{
Greiciele Farias da SILVEIRA ${ }^{1}$, Ana Aparecida Bandini ROSSI ${ }^{1 *}$, Uéliton Alves de OLIVEIRA ${ }^{1}$, \\ Tatiane Lemos VARELLA ${ }^{1}$, Fernanda Saragosa ROSSI ${ }^{1}$, Alessandro Antonio CAVALLARI ${ }^{1}$, \\ Edinéia Aparecida dos Santos GALVANIN ${ }^{2}$
}

\author{
${ }^{1}$ Faculdade de Ciências Biológicas e Agrárias, Universidade do Estado de Mato Grosso, Alta Floresta, MT, Brasil. \\ ${ }^{2}$ Faculdade de Ciências Exatas e Tecnológicas, Universidade do Estado de Mato Grosso, Barra do Bugres, MT, Brasil. \\ *E-mail: anabanrossi@unemat.br
}

Recebido em abril/2018; Aceito em agosto/2018.

\begin{abstract}
RESUMO: O objetivo do presente trabalho é caracterizar biometricamente os frutos e sementes de Passiflora cristalina Vanderplank \& Zappi ocorrentes na região de Alta Floresta, Mato Grosso. Foram coletados em 2013 um total de 100 frutos maduros de 15 genótipos e destes foram selecionadas 300 sementes aleatoriamente. Os frutos foram analisados quanto ao comprimento, largura, espessura, peso, espessura da casca, o peso da polpa, o peso das sementes/fruto, número de sementes/fruto e teor de sólidos solúveis. As sementes foram avaliadas em relação ao comprimento, largura, espessura e índice de volume. As características biométricas foram analisadas mediante distribuição de frequência calculando-se o coeficiente de correlação de Spearman e o nível de significância através do teste T. O comprimento do fruto correlacionou-se positivamente com a largura e peso do fruto, espessura da casca e com peso das sementes, não havendo uma correlação com o número de sementes por fruto. Houve uma ampla distribuição de frequência das características avaliadas nos frutos, com exceção do peso das sementes. A alta variação fenotípica encontrada para a maioria das características avaliadas nos frutos e sementes de $P$. cristalina revela que a espécie tem divergência genética a ser utilizado em programas de seleção e conservação de recursos genéticos.
\end{abstract}

Palavras-chave: divergência genética, maracujá, recursos genéticos.

\section{Biometric analysis of fruits and seeds of Passiflora cristalina Vanderplank \& Zappi}

\begin{abstract}
The present work has presented the fruits and seeds of Passiflora cristalina Vanderplank \& Zappi occurring in the region of Alta Floresta, Mato Grosso. A total of 100 mature fruits of 15 genotypes were collected in 2013 and 300 seeds were randomly selected. The fruits were inert to the length, thickness, thickness, weight, bark thickness, pulp weight, seed/fruit weight, number of seeds / fruit and soluble solids content. The seeds were evaluated for length, width, thickness and volume index. The biometric characteristics were analyzed with the calculation frequency calculated with the Spearman correlation coefficient and the level of significance throughout the $\mathrm{T}$ test. Fruit weight was correlated with fruit width and weight, bark thickness and weight of the fruits. Seeds without a key with the number of seeds per fruit. There is an offer of food distribution in the fruits, with the exception of the weight of the seeds. The high phenotypic variance found for a greater number of species evaluated in fruits and seeds of P.cristalina reveals that a type of genetics has been characterized in programs of selection and conservation of genetic resources.
\end{abstract}

Keywords: genetic divergence, passion fruit, genetic resources.

\section{INTRODUÇÃO}

A família Passifloraceae é nativa dos trópicos e subtrópicos composta por 12 gêneros, das quais 4 ocorrem no Brasil: Dikea, Tetrastylis, Mitostema e Passiflora. O gênero Passiflora é o maior da família Passifloraceae, sendo formado por aproximadamente 530 espécies tropicais e subtropicais (HANSEN et al., 2006).

Além de ser um dos gêneros mais estudados, devido aos seus sistemas de polinização ou biologia floral, bem como, pela sua importância econômica em função da qualidade dos frutos para consumo ou por apresentarem propriedades medicinais.

De forma geral, os frutos de espécies do gênero Passiflora são consumidos in natura ou utilizados para produção de sucos e doces, e muitas espécies possuem valor ornamental, em função da vistosidade e exuberância de suas flores (CERQUEIRA-SILVA et al., 2012).
Passiflora cristalina Vanderplank; Zappi (2011) é uma espécie nativa da Floresta Amazônica com ocorrência natural no Município de Alta Floresta e caracteriza-se pelo seu potencial ornamental, devido à beleza de suas flores, os frutos são utilizados como fonte de alimento por muitas espécies de animais silvestres, tais como pássaros, macacos e alguns roedores. Podendo ainda, serem utilizadas como fontes de propriedades farmacológicas ou fonte de genes de resistências para algumas espécies cultivadas, além de apresentarem propriedades nutricionais para o consumo in natura.

Apesar da importância econômica e ambiental da espécie em estudo, esta vem sofrendo com o agravamento dos problemas ambientais em nível global, como o desmatamento e as queimadas, o avanço das fronteiras agrícolas na região amazônica, em detrimento das áreas naturais. Além disto, $P$. cristalina é pouco estudada, mesmo para os parâmetros básicos das características morfológicas da planta e 
biométricas dos frutos e sementes, pois é uma espécie recentemente descrita na literatura.

Estudos morfológicos de frutos e sementes de espécies silvestres são utilizados frequentemente, visando auxiliar no conhecimento do sistema reprodutivo para futuramente serem utilizados em programas de melhoramento, subsidiando estudos de germinação, resistência, produtividade e qualidade dos frutos, auxiliando em técnicas para espécies cultivadas (BATTILANI et al., 2012).

De acordo com Marim et al. (2009) a caracterização e a avaliação é de vital importância para descrever diversos acessos por meio de características de interesse, pois a partir desses dados é possível analisar a diversidade genética dos diferentes acessos e avaliar seu potencial de uso em programas de melhoramento.

A biometria constitui um importante instrumento para detectar a variabilidade genética dentro de populações de uma mesma espécie, e as relações entre esta variabilidade e os fatores ambientais, fornecendo informações para exploração de recursos de caráter econômico e conservação (GONÇALVES et al., 2013; VIEIRA; GUSMÃO, 2008).

A existência de variabilidade para os caracteres de interesse agronômicos cujo relacionado com a planta, como os de qualidade, relacionados com o fruto, permite esperar sucesso em um programa de melhoramento (OLIVEIRA et al., 2009).

Neste contexto e considerando a escassez de estudos sobre a espécie, este trabalho teve como objetivo caracterizar biometricamente frutos e sementes de plantas de $P$. cristalina ocorrentes na região de Alta Floresta, Mato Grosso.

\section{MATERIAL E MÉTODOS}

Para a caracterização biométrica dos frutos e sementes de P. cristalina foram coletados frutos de quinze (15) plantas matrizes distribuídas em populações naturais no município de Alta Floresta - Mato Grosso, localizado no extremo norte do estado de Mato Grosso a 830 km da capital, Cuiabá (Figura 1). O município de Alta Floresta possui uma área territorial de $8.953 \mathrm{~km}^{2}$ (IBGE, 2017), e faz parte da Mesorregião Norte Mato-grossense, situado entre as coordenadas geográficas de $9^{\circ} 02^{\prime} 29^{\prime \prime}$ a $11^{\circ} 15^{\prime} 45^{\prime \prime}$ de Latitude Sul e $54^{\circ} 44^{\prime} 55^{\prime \prime}$ a $58^{\circ}$ 45' 10" de Longitude Oeste (BUTTURI et al., 2013).

No período de frutificação, entre setembro e novembro de 2013, foram coletados 100 frutos maduros de $P$. cristalina em 15 indivíduos que ocorrem naturalmente nas bordas de fragmentos florestais (Figura 1). Os frutos foram identificados, acondicionados em embalagens de papel ainda em campo e transportados para o Laboratório de Genética Vegetal e Biologia molecular da Universidade do Estado de Mato Grosso, campus de Alta Floresta-MT, onde se procedeu a caracterização.

Os frutos foram avaliados, mediante as seguintes características: comprimento do fruto $(\mathrm{CF})$; largura do fruto (LF); espessura da casca fresca (ECF) e espessura do fruto (EF), ambas as medidas foram obtidos com auxílio de um paquímetro digital Mitutoyo, com precisão de $0,01 \mathrm{~mm}$. Foi avaliado também o peso do fruto fresco (PFF) e da polpa fresca (PPF) obtidos através de uma balança de precisão de 0,00001 g. O peso da polpa foi determinado para cada fruto, subtraindose o peso das sementes/fruto do peso da polpa + sementes/fruto.

Após a caracterização, os frutos foram seccionados e obteve-se o teor de sólidos solúveis (BRIX) através de um refratômetro portátil, com leitura na faixa de 0 a $32^{\circ}$, segundo o trabalho proposto por Pereira et al., (2006).

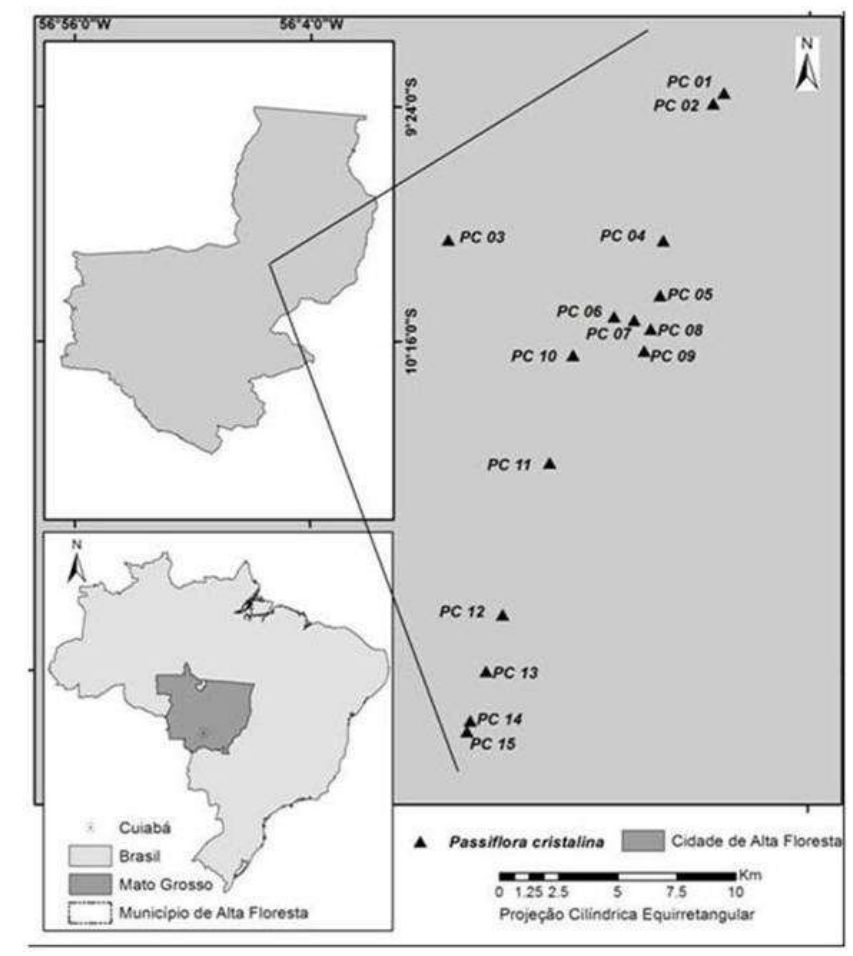

Figura 1. Mapa da área de estudo. Com destaque para o município de Alta Floresta, MT e a localização dos 15 genótipos de Passiflora cristalina.

Figure 1. Map of the study area. With emphasis on the municipality of Alta Floresta, MT and the location of the 15 genotypes of Passiflora cristalina.

Posteriormente as sementes foram retiradas dos frutos e lavadas em água corrente, para remoção do arilo das sementes foram realizadas fricções manuais em peneira de malha plástica. Logo em seguida, foi avaliado o número de sementes por fruto (NSF) e o peso das sementes por fruto (PSF).

Em cada semente foi mensurado, com um paquímetro digital $(0,01 \mathrm{~mm})$, o comprimento longitudinal (CS), a largura mediana (LS) e a espessura mediana (ES). Determinou-se o índice de volume das sementes (IVS) através do produto do comprimento $\mathrm{x}$ largura $\mathrm{x}$ espessura, para estimativa dos tamanhos e comparações.

As características biométricas dos frutos e sementes foram analisadas mediante distribuição de frequência. Os dados obtidos não aderem à distribuição normal, logo se utilizou o coeficiente de correlação não paramétrico de Spearman (rS) e o respectivo nível de significância (p) entre as variáveis através do Teste T (ZAR, 1996). Todas as análises estatísticas foram realizadas por meio da versão DEMO do programa BIOESTAT 5.3 (AYRES et al., 2007).

\section{RESULTADOS}

Os frutos de Passiflora cristalina possuem formato ovóide, do tipo baga, apresentando coloração esverdeada com máculas brancas, adquirindo coloração marrom quando maduros e sabor agridoce (Figura 2). Os resultados da análise descritiva geral dos frutos estão apresentados na Tabela 1. Observa-se que ocorreu uma ampla variação entre os valores mínimos e máximos para todas as características avaliadas nos frutos de P. cristalina. A média e a mediana apresentaram valores bem próximos, enquanto a assimetria e curtose apresentaram 
valores próximos de zero, o que pode indicar uma aproximação da distribuição normal, exceto, para a variável espessura da casca e peso das sementes.
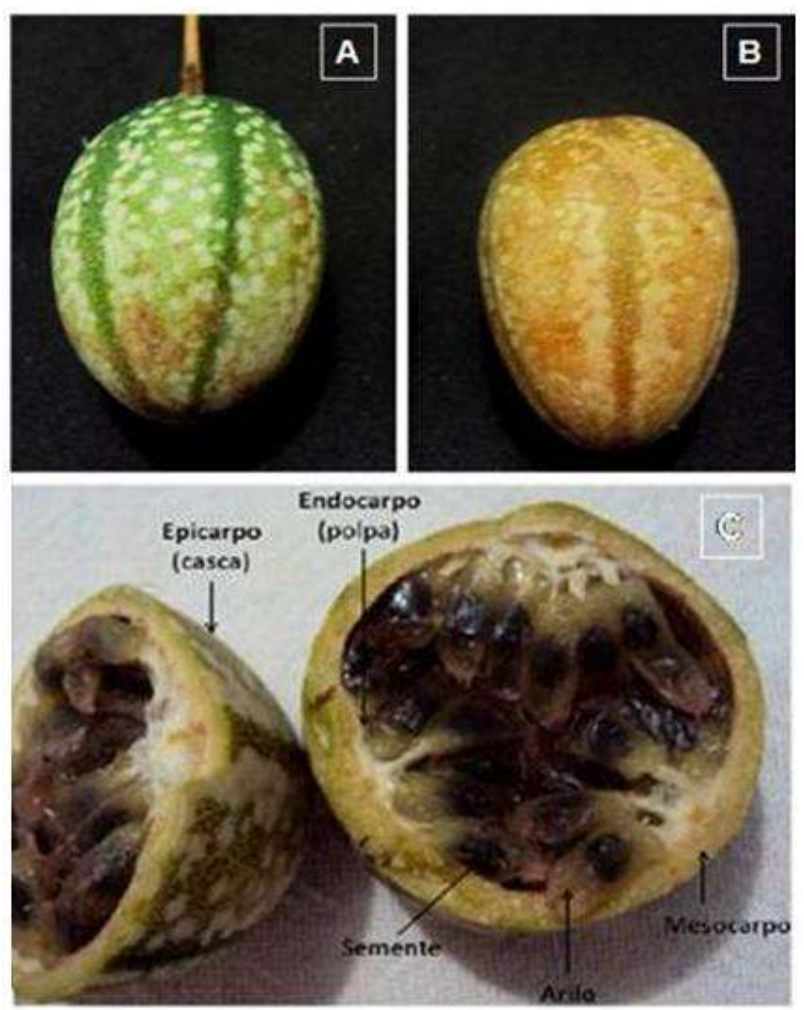

Figura 2. Detalhe dos frutos de Passiflora cristalina. A: Fruto verde; B: Fruto maduro; C: Detalhes da parte interna do fruto maduro (FREITAS, 2010).

Figure 2. Detail of the fruits of Passiflora cristalina. A: Green fruit; $\mathrm{B}$ : Mature fruit; C: Details of the internal part of the ripe fruit (FREITAS, 2010).

O comprimento dos frutos apresentou a média de 44,14 $\mathrm{mm}$, distribuindo-se principalmente em duas classes, de 44,68 a $47,80 \mathrm{~mm}(23 \%)$ e de 47,80 a $50,93 \mathrm{~mm}(26 \%)$, totalizando $49 \%$ dos frutos amostrados (Figura $3 \mathrm{~A}$ ).

Cerca de $32 \%$ dos frutos, apresentou largura de 36,99 a $39,79 \mathrm{~mm}$ com limite mínimo e máximo de 25,79 e 42,59 mm (Figura 3B e Tabela 01) e a espessura do fruto obteve, uma média de $32,97 \mathrm{~mm}$, sendo que $36 \%$ do total de frutos avaliados, ficaram alocados na classe 31,67 a $34,84 \mathrm{~mm}$ de espessura (Figura 3C). A casca do fruto apresentou uma espessura média de $2,04 \mathrm{~mm}$, com maioria dos frutos $(44 \%)$ concentrados na classe de 1,77 a 2,08 mm (Figura 3D).
O peso do fruto apresentou um limite mínimo de 12,66 g e um máximo de 43,16 g, com média de 27,71 g (Tabela 1), sendo que cerca de $30 \%$ dos frutos ficaram concentrados na classe de 27,91 a 32,99 g (Figura 3E). Já o peso da polpa distribuiu-se em duas classes que representaram cerca de $51 \%$ dos frutos amostrados, reunidos na classe de 15,00 a 18,20 g (26\%) e na classe de 18,20 a 21,50 g (25\%) (Figura 3F). O peso das sementes por fruto apresentou um limite mínimo de $0,99 \mathrm{~g}$ e um limite máximo de $9,30 \mathrm{~g}$, sendo que a maioria as sementes ficaram concentrada na classe 0,99 a 2,38 g, o que representou cerca de $60 \%$ das sementes avaliadas (Figura $3 \mathrm{G}$ ).

Quanto ao número de sementes por fruto (Figura $3 \mathrm{H}$ ), observou-se uma ampla distribuição de frequência entre as classes, sendo comumente encontrado em $27 \%$ dos frutos avaliados de 180 a 218 e $26 \%$ na classe de 218 a 256 sementes. Sendo que mais $90 \%$ dos frutos apresentaram uma quantidade superior a 100 sementes, característica de grande importância para reprodução da espécie.

As sementes podem ser consideradas como a principal forma de propagar as espécies, bem como de propiciar a sobrevivência das plantas em condições adversas. Portanto, quanto maior o número de sementes por fruto em $P$. cristalina maior a probabilidade de deixar descendentes.

O teor de sólidos solúveis apresentou um limite mínimo de $5^{\circ}$ BRIX e um máximo de $26^{\circ}$ BRIX e distribuiu-se em duas classes, sendo uma classe de frutos concentrando $31 \%$ dos frutos e a outra classe com cerca de $28 \%$, totalizando $59 \%$ (Figura 4). As sementes de $P$. cristalina (Figura 5) são ovais, achatadas, com texturas proeminentes, cor escura e são recobertas por uma polpa sucosa e amarelada (FREITAS, 2010).

A análise descritiva geral, para as variáveis analisada nas sementes de $P$. cristalina, está apresentada na Tabela 2 e Figura 6. Os valores de média e mediana assemelharam-se a assimetria e curtose, apresentando valores próximos de zero, indicando que apenas algumas variáveis apresentaram valores próximos à distribuição normal.

A distribuição de frequência do comprimento das sementes concentrou cerca de 55,33\% das sementes (Figura 6A). Da mesma forma a largura das sementes concentrou-se em uma única classe de com 64,67\% (Figura 6B).

Quanto à espessura, as sementes ficaram alocadas em duas classes que se distribuíram entre 0,71 a 1,22 mm (45,67\%) e 1,22 a $1,73(49,33 \%)$ com aproximadamente $95 \%$ das sementes alocadas nestas classes (Figura 6C). A classe de 19,58 a $23,06 \%$ concentrou $24,33 \%$ do total do índice de volume das sementes (Figura 6D).

Tabela 1. Caracterização biométrica dos frutos e sementes de Passiflora cristalina. Table 1. Biometric characterization of fruits and seeds of Passiflora cristalina.

\begin{tabular}{lllllllll}
\hline Parâmetro & Mínimo & Média & Máximo & Mediana & Assimetria & Curtose & DP & CV\% \\
\hline CF (mm) & 35,30 & 44,14 & 53,95 & 44,82 & $-0,17$ & $-1,07$ & 4,67 & 10,58 \\
LF (mm) & 25,79 & 36,59 & 42,59 & 37,08 & $-0,68$ & 0,17 & 3,50 & 9,56 \\
EF (mm) & 22,14 & 32,97 & 41,09 & 33,24 & $-0,25$ & $-0,15$ & 4,29 & 13,01 \\
ECF (mm) & 1,45 & 2,04 & 3,24 & 1,96 & 1,16 & 1,56 & 0,39 & 19,31 \\
PFF (g) & 12,66 & 27,71 & 43,06 & 28,31 & $-0,05$ & $-0,68$ & 7,40 & 26,72 \\
PPF (g) & 5,18 & 15,33 & 23,76 & 15,85 & 0,24 & 0,84 & 1,41 & 30,32 \\
PSE (g) & 0,99 & 2,36 & 9,2 & 2,22 & 3,38 & 21,10 & 1,00 & 42,40 \\
NSF (g) & 65 & 188,01 & 293 & 198,5 & $-0,38$ & $-0,65$ & 57,05 & 30,34 \\
BRIX (\%) & 5 & 15,97 & 25 & 16,25 & $-0,27$ & $-0,65$ & 4,28 & 26,83 \\
\hline
\end{tabular}

CF: Comprimento do fruto; LF: Largura do fruto; EF: Espessura do fruto; ECF: Espessura da casca fresca; PFF: Peso do fruto fresco; PPF: Peso da polpa fresca; PSE: Peso das sementes por fruto; NSF: Número de sementes por fruto. DP - Desvio Padrão; CV - Coeficiente de variação. 
Silveira et al.

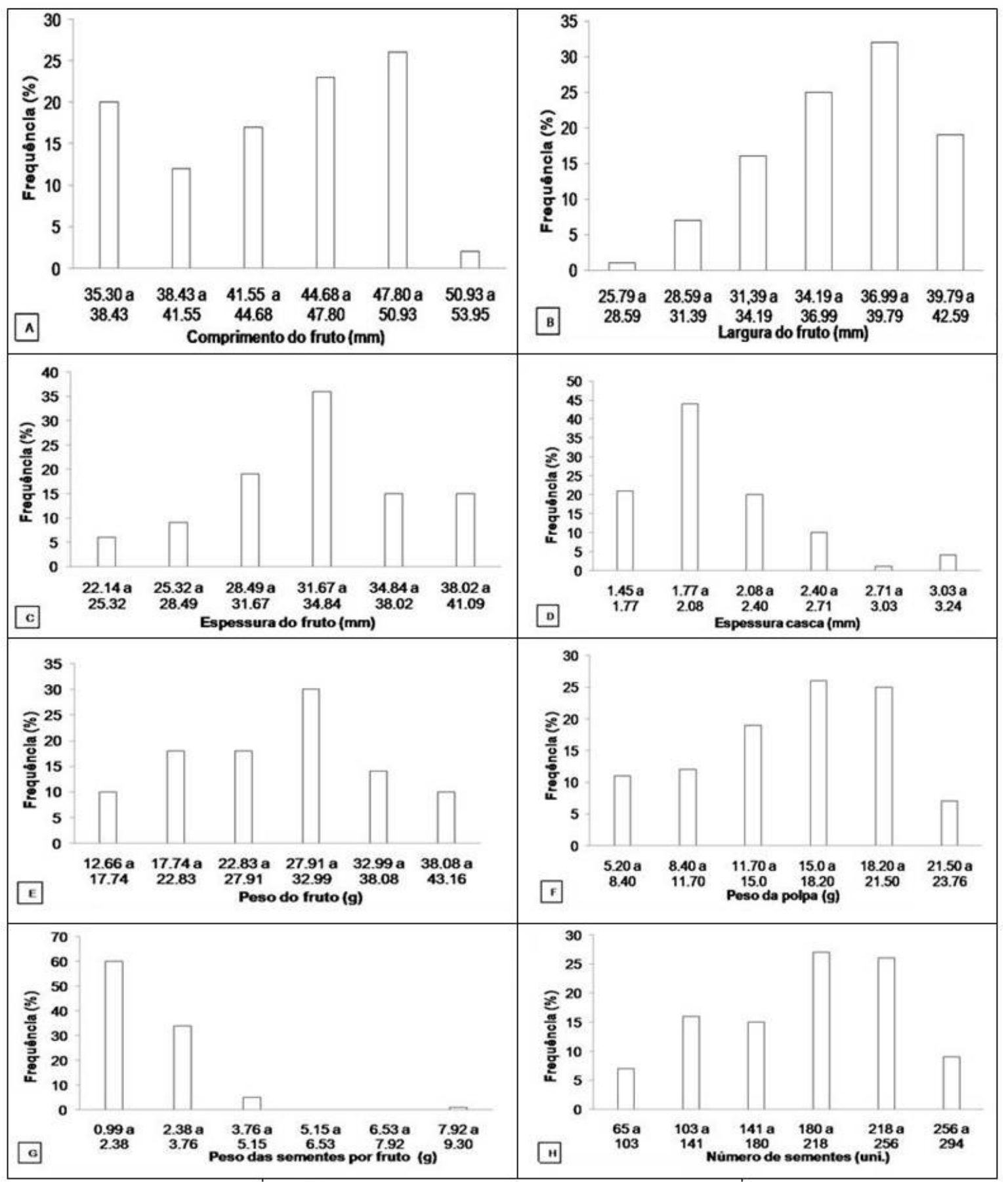

Figura 3. Distribuição de frequência com base em características biométricas dos frutos de Passiflora cristalina. Figure 3. Frequency distribution based on biometric characteristics of the fruits of Passiflora cristalina.

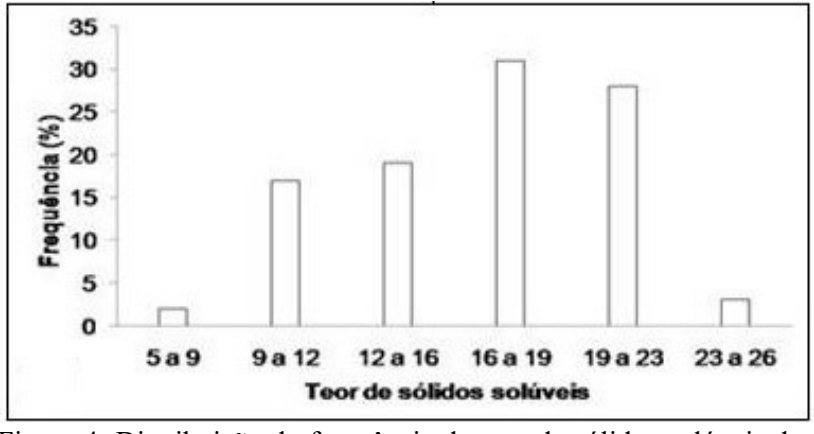

Figura 4. Distribuição de frequência do teor de sólidos solúveis dos frutos de Passiflora cristalina.

Figure 4. Frequency distribution of soluble solids content of fruits of Passiflora cristalina.

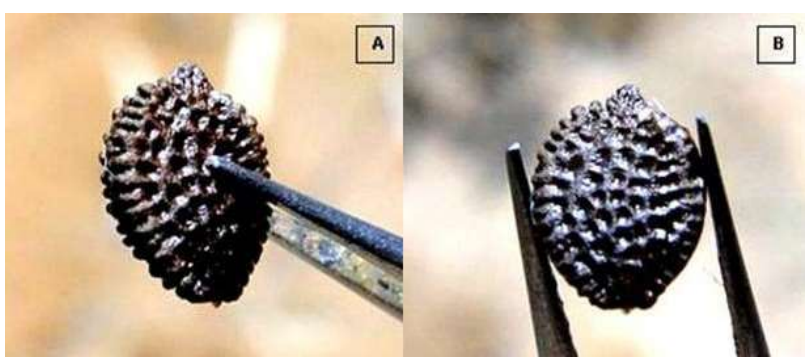

Figura 5. Detalhes das Sementes de Passiflora cristalina (FREITAS, 2010).

Figure 5. Details of the seeds of Passiflora cristalina (FREITAS, 2010). 
As estimativas dos coeficientes de correlação de Spearman (rS) entre as características biométricas de frutos e sementes de $P$. cristalina encontram-se na Tabela 3. A correlação de Spearman demonstrou que o comprimento dos frutos se correlacionou positivamente com a largura, espessura da casca, peso do fruto e com peso das sementes.

A largura do fruto se integrou com a espessura do fruto, espessura da casca, peso do fruto e peso das sementes.

A espessura do fruto correlacionou-se positivamente com espessura da casca, peso do fruto, e peso das sementes. Prontamente a espessura da casca associou-se com o peso do fruto, peso da polpa e peso das sementes.

Sendo assim, tanto a espessura da casca, quanto a espessura do fruto está correlacionada com o peso das sementes. Quanto maior o número de sementes, maior será o peso do fruto e peso das sementes, e consequentemente maior será o rendimento da polpa, uma vez que cada semente está envolta pelo arilo.

Houve correlação entre o peso do fruto e o peso da polpa com o peso das sementes por fruto. Assim, à maior quantidade de polpa está associada ao maior peso das sementes.

Não foi observada nenhuma correlação dos frutos com as características biométricas das sementes, exceto para as próprias características avaliadas na semente. Houve correlação entre a espessura e a largura e o índice de volume das sementes, $0,60 * *$ e $0,85^{* *}$, respectivamente. Foi observada também uma correlação entre o comprimento e espessura da semente.

Tabela 2. Análise descritiva das sementes frescas de Passiflora cristalina. CS: Comprimento da semente; LS: Largura da semente; ES: Espessura da semente; IVS: Índice de volume das sementes.

Table 2. Descriptive analysis of fresh seeds of Passiflora cristalina. CS: Seed length; LS: Seed width; ES: Seed thickness; IVS: Seed volume index.

\begin{tabular}{lllllllll}
\hline Parâmetro & Mínimo & Média & Mediana & Máximo & Assimetria & Curtose & DP & CV $(\%)$ \\
\hline CS $(\mathrm{mm})$ & 3,85 & 4,92 & 4,94 & 5,53 & $-0,94$ & 2,94 & 0,22 & 4,59 \\
LS $(\mathrm{mm})$ & 2,08 & 3,24 & 3,25 & 3,83 & $-1,63$ & 11,18 & 0,16 & 4,92 \\
ES $(\mathrm{mm})$ & 0,71 & 1,28 & 1,12 & 1,83 & 1,25 & 5,75 & 0,12 & 10,63 \\
IVS $\left(\mathrm{mm}^{3}\right)$ & 9,13 & 18,19 & 18,00 & 29,92 & 0,63 & 2,64 & 2,62 & 14,41 \\
\hline
\end{tabular}

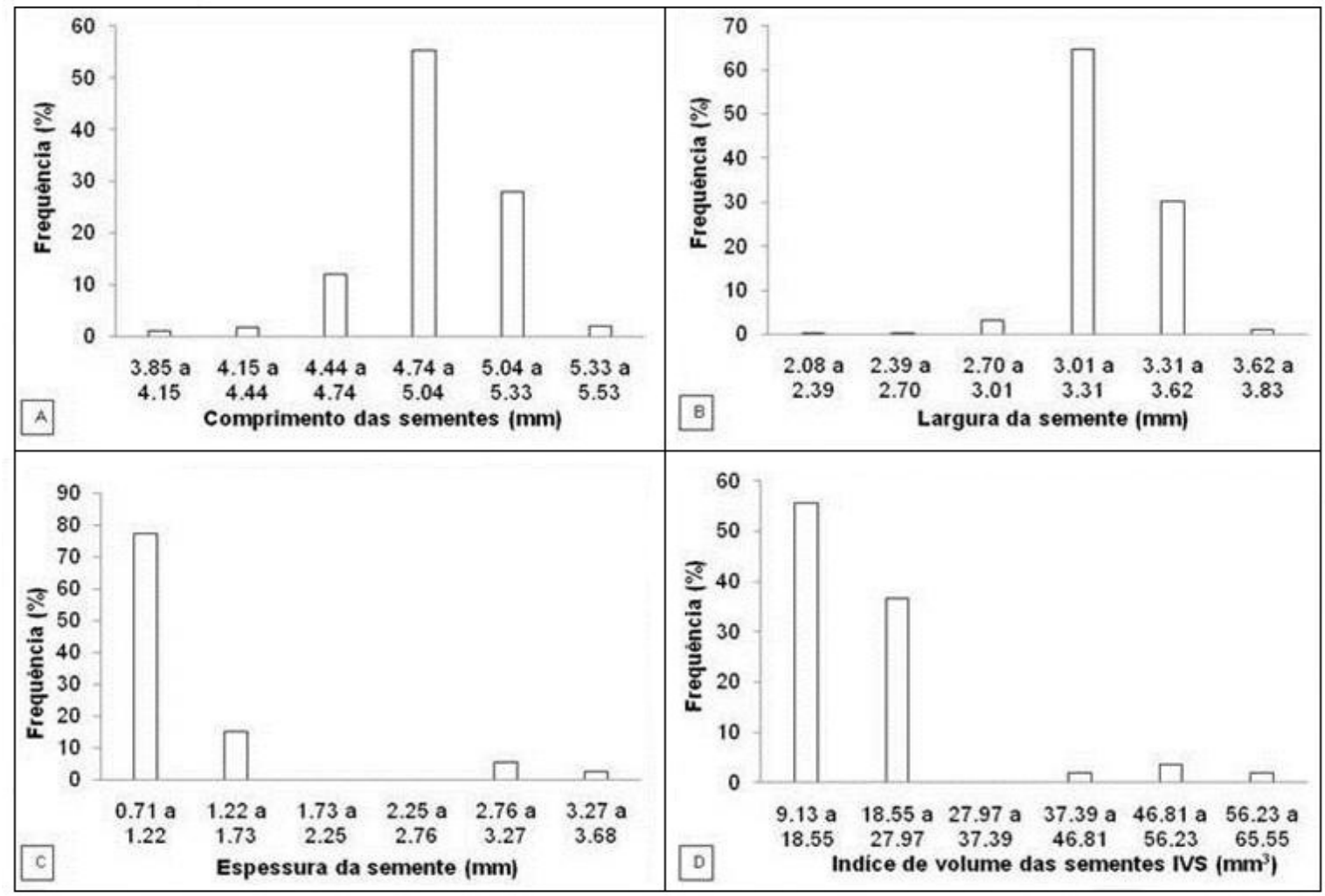

Figura 6. Distribuição de frequência com base em características biométricas das sementes de Passiflora cristalina. Figure 6. Frequency distribution based on biometric characteristics of the seeds of Passiflora cristalina.

\section{DISCUSSÃO}

Um fator de suma importância, é que o rendimento em suco está relacionado com o número de óvulos fecundados, os quais serão transformados em sementes envolvidas por um arilo ou sarcotesta e que, por sua vez, encerram o suco propriamente dito. Portanto quanto maior o número de sementes maior será o rendimento de suco por fruto (SILVA; SÃO JOSÉ, 1994).
De acordo Teixeira (1994), para a industrialização, os frutos de maracujá devem apresentar um alto teor de sólidos solúveis, maior que $15^{\circ}$ BRIX. Sendo assim, a $P$. cristalina ultrapassou a média de BRIX exigida pelas indústrias, com cerca de $59 \%$ dos frutos concentrado nas classes de 16 a $23^{\circ}$ BRIX, indicando que a espécie apresenta características promissoras, podendo ser utilizada em futuros programas de 
melhoramento genético, que visem melhorias desta característica.

Para o mercado in natura, há preferência por frutos de alto rendimento em suco e com maior teor de sólidos solúveis totais, visto que isso diminuiria a adição de adoçantes e acidificantes no suco, no entanto, são preferidos frutos mais doces e menos ácidos (CAVICHIOLI et al., 2011).

A variação fenotípica encontrada nos frutos de $P$. cristalina pode estar ligada à variabilidade genética das populações naturais analisadas, relacionada diretamente á autoimcompatibilidade do gênero (MELETTI; BRUCKNER, 2001). Este conhecimento possibilita ao melhorista explorar a diversidade, podendo realizar a introgressão de alelos favoráveis encontrados em espécies silvestres, como resistência a doenças, autocompatibilidade e redução do androginóforo, por intermédio de cruzamento interespecífico (PAIVA et al., 2014).
Vários trabalhos realizados com maracujazeiro amarelo apresentaram resultados semelhantes aos observados para esta característica, entre eles, estão os estudos desenvolvidos por Gonçalves et al. (2008); Negreiros et al. (2008); Oliveira et al. (2008); Silva et al. (2009).

A biometria de sementes e frutos fornece informações para a conservação e exploração da espécie, permitindo incremento contínuo da busca racional, uso eficaz e sustentável, sendo importantes para a seleção, pois, podem incrementar ou uniformizar estas qualidades (GONÇALVES et al., 2013). Além disso, constitui um instrumento importante para detectar a variabilidade genética dentro de populações de uma mesma espécie, e as relações entre essa variabilidade e os fatores ambientais (CARVALHO et al., 2003). As informações obtidas neste estudo servem como base pra auxiliar na escolha de genitores que possuam características de interesse comercial e resistência, e que futuramente possam ser utilizadas programas de melhoramento genético.

Tabela 3. Correlação de Spearman para caracterização biométrica dos frutos de Passiflora cristalina. CF: Comprimento do fruto; LF: Largura do fruto; EF: Espessura do fruto; ECF: Espessura da casca fresca; PFF: Peso do fruto fresco; PPF: Peso da polpa fresca; PSE: Peso das sementes por fruto; NSF: Número de sementes por fruto; CS: Comprimento da semente; LS: Largura da semente; ES: Espessura da semente; IVS: Índice de volume das sementes.

Table 3. Spearman correlation for biometric characterization of Passiflora cristalina fruits. CF: Length of fruit; LF: Width of the fruit; EF: Fruit thickness; ECF: Fresh shell thickness; PFF: Fresh fruit weight; PPF: Fresh pulp weight; PSE: Weight of seeds per fruit; NSF: Number of seeds per fruit; CS: Seed length; LS: Seed width; ES: Seed thickness; IVS: Seed volume index.

\begin{tabular}{|c|c|c|c|c|c|c|c|c|c|c|c|c|c|}
\hline & $\mathrm{CF}$ & LF & $\mathrm{EF}$ & ECF & PFF & PPF & PSE & NSF & BRIX & CSF & LSF & ESF & IVS \\
\hline $\mathrm{CF}$ & 1 & & & & & & & & & & & & \\
\hline LF & $0.51^{* *}$ & 1 & & & & & & & & & & & \\
\hline $\mathrm{EF}$ & $0.31^{\mathrm{ns}}$ & $0.71 * *$ & 1 & & & & & & & & & & \\
\hline ECF & $0.61 * *$ & $0.71 * *$ & $0.60 * *$ & 1 & & & & & & & & & \\
\hline PFF & $0.64 * *$ & $0.68^{* *}$ & $0.56^{* *}$ & $0.85^{* *}$ & 1 & & & & & & & & \\
\hline PPF & $0.12^{\text {ns }}$ & $0.21^{\mathrm{ns}}$ & $0.20^{\mathrm{ns}}$ & $0.38^{* *}$ & $0.27^{\mathrm{ns}}$ & 1 & & & & & & & \\
\hline PSE & $0.37^{*}$ & $0.58 * *$ & $0.45^{* *}$ & $0.68^{* *}$ & $0.70^{* *}$ & $0.37 *$ & 1 & & & & & & \\
\hline NSF & $0.15^{\text {ns }}$ & $0.23^{\mathrm{ns}}$ & $0.27^{\mathrm{ns}}$ & $0.32^{\mathrm{ns}}$ & $0.26^{\mathrm{ns}}$ & $0.12^{\mathrm{ns}}$ & $0.14^{\mathrm{ns}}$ & & & & & & \\
\hline BRIX & $0.09^{\mathrm{ns}}$ & $0.00^{\mathrm{ns}}$ & $0.16^{\mathrm{ns}}$ & $0.19^{\mathrm{ns}}$ & $0.25^{\mathrm{ns}}$ & $0.11^{\mathrm{ns}}$ & $0.17^{\mathrm{ns}}$ & $-0.03^{\text {ns }}$ & 1 & & & & \\
\hline CS & $-0.13^{\text {ns }}$ & $0.09^{\text {ns }}$ & $0.06^{\mathrm{ns}}$ & $0.01^{\mathrm{ns}}$ & $0.07^{\mathrm{ns}}$ & $-0.07^{\mathrm{ns}}$ & $0.00^{\mathrm{ns}}$ & $-0.11^{\mathrm{ns}}$ & -0.07 & 1 & & & \\
\hline LS & $-0.12^{\text {ns }}$ & $0.12^{\mathrm{ns}}$ & $0.09^{\mathrm{ns}}$ & $-0.02^{\text {ns }}$ & $0.03^{\text {ns }}$ & $-0.06^{\mathrm{ns}}$ & $-0.02^{\mathrm{ns}}$ & $-0.01^{\mathrm{ns}}$ & $-0.06^{\mathrm{ns}}$ & $0.25^{\mathrm{ns}}$ & 1 & & \\
\hline ES & $-0.11^{\mathrm{ns}}$ & $0.04^{\mathrm{ns}}$ & $0.06^{\mathrm{ns}}$ & $-0.03^{\text {ns }}$ & $0.06^{\mathrm{ns}}$ & $0.02^{\mathrm{ns}}$ & $0.02^{\mathrm{ns}}$ & $0.06^{\mathrm{ns}}$ & $-0.06^{\mathrm{ns}}$ & $0.12 * *$ & $0.21^{\mathrm{ns}}$ & 1 & \\
\hline IVS & $-0.17^{\mathrm{ns}}$ & $0.09^{\text {ns }}$ & $0.09^{\text {ns }}$ & $-0.03^{\text {ns }}$ & $0.07^{\mathrm{ns}}$ & $-0.03^{\mathrm{ns}}$ & $-0.00^{\text {ns }}$ & $-0.01^{\mathrm{ns}}$ & $-0.11^{\mathrm{ns}}$ & $0.48^{\mathrm{ns}}$ & $0.60 * *$ & $0.85^{* *}$ & 1 \\
\hline
\end{tabular}

*significativo a $5 \%$ de probabilidade; ** significativo a $1 \%$ de probabilidade; ns não significativo.

\section{CONCLUSÕES}

A grande variação nas características biométricas dos frutos de Passiflora cristalina pode ser resultado da expressão gênica da variabilidade genética existente nas populações naturais associadas às condições ambientais locais. Ocorreu uma ampla distribuição de frequência entre as classes das características avaliadas nos frutos, com exceção do peso das sementes que se concentrou nas duas primeiras classes. A maioria dos frutos apresentou acima de 100 sementes, característica importante para manutenção da espécie no ambiente, pois quanto maior o número de sementes por fruto maior a probabilidade de deixar descendentes.

\section{AGRADECIMENTOS}

À Universidade do estado de Mato Grosso, a equipe do Laboratório de Genética vegetal e Biologia molecular e as instituições de pesquisa: Fundação de amparo à pesquisa do estado do Mato Grosso (FAPEMAT) e Coordenação de Aperfeiçoamento de Pessoal de Nível Superior (CAPES) pela concessão de bolsa a primeira autora.

\section{REFERÊNCIAS}

AYRES, M.; AYRES-JÚNIOR, M.; AYRES, D. L.; SANTOS, A. S. BioEstat 5.0: aplicações estatísticas nas áreas das ciências biológicas e médicas. Belém: MCT; IDSM; CNPq. 2007. 364 p.

BATTILANI, J. L.; SANTIAGO, E. F.; DIAS, E. S. Morfologia de frutos, sementes, plântulas e plantas jovens de Guibourtia hymenifolia (Moric.) J. Leonard (Fabaceae). Revista Árvore, Viçosa, v. 35, n. 5, p. 1089-1098, mai. 2012. DOI: http://dx.doi.org/10.1590/S010067622011000600015

BUTTURI, W.; NUNES, E. J. S.; SILVA, E. P. Banco de dados geográfico aplicado ao cadastro ambiental rural do município de Alta Floresta - MT. Revista de Ciências Agro-Ambientais, Alta Floresta, v .11, n.1, p.1-8, mar. 2013.

CAVICHIOLI, J. C.; CORREAA, L. S.; BOLIANI, A. C.; SANTOS, P. C. Características físicas e químicas de frutos de maracujazeiro amarelo enxertado em três portaenxertos. Revista Brasileira de Fruticultura, 
Jaboticabal, v. 33, n. 3, p. 905-914, set. 2011. DOI: http://dx.doi.org/10.1590/S0100-29452011000300026

CARVALHO, J. E. U.; NAZARÉ, R. F. R.; OLIVEIRA, W. M. Características físicas e físico-químicas de um tipo de bacuri (Platonia insignis Mart.) com rendimento industrial superior. Revista Brasileira de Fruticultura, Cruz das Almas, v. 25, n. 2, p. 326-328, abr. 2003. DOI: http://dx.doi.org/10.1590/S0100-29452003000200036

CERQUEIRA-SILVA, C. B. M.; SANTOS, E. S. L.; CONCEIÇÃO, L. D.; CARDOSO-SILVA, C. B.; PEREIRA, A. S.; OLIVEIRA, A. C.; CORRÊA, R. X. Genetic variation in a wild population of the 'sleep' passion fruit (Passiflora setacea) based on molecular markers. Genetics and Molecular Research. Ribeirão Preto, v. 11, n. 1, p.731-738, mar. 2012. DOI: https://dx.doi.org/10.4238/2012.March.22.3

FREITAS, E. J. Biologia Reprodutiva e germinação de Passiflora cristalina Vanderpl. \& Zappi em fragmentos florestais no perímetro urbano do município de Alta Floresta-MT. 2010. 73f. Trabalho de conclusão de curso (Bacharelado). Universidade do Estado de Mato Grosso. Alta Floresta, 2010.

GONÇALVES, G. M.; VIANA, A. P.; REIS, LUCILÉIA. S.; BEZERRA NETO, F. V.; AMARAL JÚNIOR, A. T.; LAUDIRLÉA, S. Correlações fenotípicas e genéticoaditivas em maracujá-amarelo pelo delineamento I. Ciência Agrotécnica, Lavras, v. 32, n. 5, p. 1413-1418, out. 2008. DOI: https://dx.doi.org/10.1590/S141370542008000500009

GONÇALVES, L. G. V.; ANDRADE, F. R.; MARIMON JUNIOR, B. H.; SCHOSSLER, T. R.; LENZA, E.; MARIMON, B. S. Biometria de frutos e sementes de mangaba (Hancornia speciosa Gomes) em vegetação natural na região leste de Mato Grosso, Brasil. Revista de Ciências Agrárias, Lisboa, v. 36, n. 1, p. 31-40, jan. 2013.

HANSEN, A. K.; GILBERT, L. E.; SIMPSON, B. B.; CERVI, A. C.; JANSEN, R. K. Phylogenetic relationships and chromosome number evolution in Passiflora. Systematic Botany, Kent, v. 31, n. 1, p. 38-150, jan. 2006. DOI: http://dx.doi.org/10.1600/036364406775971769

IBGE_INSTITUTO BRASILEIRO DE GEOGRAFIA E ESTATÍSTICA. Censo 2017. 2017. Disponível em: $<$ https://cidades.ibge.gov.br/brasil/mt/altafloresta/panora ma>. Acesso em 13 abr. 2018.

MARIM, B. G.; SILVA, D. J. H.; CARNEIRO, P. C. S.; MIRANDA, G. V. M.; MATTEDI, A. P.; CALIMAN, F. R. B. Variabilidade genética e importância relativa de caracteres em acessos de germoplasma de tomateiro. Pesquisa agropecuária Brasileira, Brasília, v. 44, n. 10, p. 1283-1290, set. 2009.

MELETTI, L. M. M.; BRUCKNER, C. H. 2001. Melhoramento genético. In: BRUCKNER, C. H, PICANÇO, M. C (Ed.). Maracujá: tecnologia de produção, pós-colheita, agroindústria, mercado. Porto Alegre: Cinco continentes. p. 345-385.

NEGREIROS, J. R. S.; ARAÚJO NETO, S. E.; ÁLVARES, V. S.; LIMA, V. A.; OLIVEIRA, T. K. Caracterização de frutos de progênies de meios-irmãos de maracujazeiro amarelo em Rio Branco - Acre. Revista Brasileira de Fruticultura, Jaboticabal, v. 30, n. 2, p. 431-437, jun. 2008. DOI: http://dx.doi.org/10.1590/S010029452008000200028
OLIVEIRA, E. J.; SANTOS, V. S.; LIMA, D. S.; MACHADO, M. D.; LUCENA, R. S.; MOTTA, T. B. N.; CASTELLEN, M. S. Seleção em progênies de maracujazeiro amarelo com base em índices multivariados. Pesquisa Agropecuária Brasileira, Brasília, v. 43, n. 11, p. 1543-1549, nov. 2008. DOI: http://dx.doi.org/10.1590/S0100-204X2008001100013

OLIVEIRA, M. E. B.; GUERRA, N. B.; MAIA, A. de H. N.; ALVES, R. E.; XAVIER, D. da S.; MATOS, N. M. dos S. Caracterização física de frutos do pequizeiro nativos da chapada do Araripe. Revista Brasileira de Fruticultura, Jaboticabal, v. 31, n. 4, p. 1196-1201, dez. 2009. DOI: http://dx.doi.org/10.1590/S0100-29452009000400038

PAIVA, C. L.; VIANA, A. P.; SANTOS, E. A.; SILVA, R. N. O.; OLIVEIRA, E. J. Diversidade genética de espécies do gênero Passiflora com o uso da estratégia ward-mlm ${ }^{1}$. Revista Brasileira de Fruticultura, Jaboticabal, v. 36, n. 2, p. 381-390, jun. 2014. DOI: http://dx.doi.org/10.1590/0100-2945-156/13

PEREIRA; M. E. C.; CANTILLANO F. F.; GUTIEREZ, A. S. D.; ALMEIDA G. V. B. Procedimentos pós-colheita na produção integrada de citros. Cruz das Almas: Embrapa Mandioca e Fruticultura Tropical, 2006. 40 p. (Documentos, 156)

SILVA, A. C.; SÃO JOSÉ, A. R. Classificação botânica do maracujazeiro. In: SÃO JOSÉ, A. R (Ed.). Maracujá: produção e mercado. Vitória da Conquista: Universidade Estadual do Sudoeste da Bahia, 1994. p.1-5.

SILVA, L. J. B. da.; SOUZA, M. L. de.; ARAÚJO NETO, S. E. de.; MORAIS, A. P. Revestimentos alternativos na conservação pós-colheita de maracujá- amarelo. Revista Brasileira de Fruticultura, Jaboticabal, v. 31, n. 4, p. 995-1003, dez. 2009. DOI: http://dx.doi.org/10.1590/S0100-29452009000400012

TEIXEIRA, C. G. Maracujá: cultura. In: Instituto de tecnologia de alimentos. Maracujá: cultura, matériaprima, processamento e aspectos econômicos. 2. rd. Campinas: Secretaria da Agricultura e Abastecimento/ITAL, 1994. p. 3-131.

VANDERPLANK. J.; ZAPPI, D. Passiflora cristalina, a striking new species of Passiflora (Passifloraceae) from Mato Grosso, Brazil. Kew Bulletin, London, v. 66, n. 1, p. 149-153, mar. 2011.2 DOI: https://dx.doi.org/10.1007/s12225-011-9255-2

VIEIRA, F. A.; GUSMÃO, E. Biometria, armazenamento de sementes e emergência de plântulas de Talisia esculenta Radlk. (Sapindaceae). Ciência e Agrotecnolgia, Lavras, v. 32, n. 4, p. 1073-1079, ago. 2008. DOI: https://dx.doi.org/10.1590/S1413-70542008000400006

ZAR, J. H. Biostatistical analysis. 3. ed. New York: PrenticeHall. 1996. 662 p. 